\title{
miR-488-5p promotes esophageal squamous cell carcinoma progression by suppressing the P53 pathway
}

\author{
Chang Su ${ }^{1,2}$, Wenxiu Liu ${ }^{3}$, Tao Jiang ${ }^{1}$, Junfeng Liu ${ }^{1}$ \\ ${ }^{1}$ Department of Thoracic Surgery, Fourth Hospital, Hebei Medical University, Shijiazhuang, China; ${ }^{2}$ Department of Cardiothoracic Surgery, \\ Bethune International Peace Hospital, Shijiazhuang, China; ${ }^{3}$ Department of Cardiology, Bethune International Peace Hospital, Shijiazhuang, China \\ Contributions: (I) Conception and design: C Su; (II) Administrative support: J Liu; (III) Provision of study materials or patients: C Su; (IV) Collection \\ and assembly of data: W Liu; (V) Data analysis and interpretation: T Jiang; (VI) Manuscript writing: All authors; (VII) Final approval of manuscript: \\ All authors. \\ Correspondence to: Junfeng Liu. Department of Thoracic Surgery, Fourth Hospital, Hebei Medical University, Shijiazhuang 050011, China. \\ Email: 13931152296@163.com.
}

Background: miR-488-3p has been reported to play an important role in cancer progression and metastasis. The protein 53 (P53) gene serves as a mediator and biomarker of esophageal squamous cell carcinoma (ESCC). However, the molecular mechanism underlying miR-488-5p in the pathology of ESCC through the P53 pathway has not been examined.

Methods: The expression levels of miR-488-5p were determined by quantitative reverse transcription polymerase chain reaction (qRT-PCR). Cytological experiments were performed to evaluate the biological functions of miR-488-5p. A bioinformatics analysis was performed to determine the pathways and key miR488-5p targets associated with ESCC. Correlations between miR-488-5p and P53 signaling pathways were validated by western blotting and the dual luciferase reporter gene system. Finally, the expression level of miR-488-5p was regulated and tumor formation experiments were performed in nude mice.

Results: The qRT-PCR analysis showed that MiR-488-5p expression was more upregulated in the KYSE150 group than the HEEC group. In the KYSE-150 cells, the colony formation assay and flow cytometry analysis indicated that the miR-488-5p inhibitor inhibited cell viability and increased cell apoptosis; however, these effects were recovered by P53 knockdown (KD). In addition, cell invasion and cell migration were inhibited by the miR-488-5p inhibitor, but were also improved by P53 KD. Similarly, the miR-488-5p inhibitor induced the expression of P53 and P21 than normal control (NC) group in which miR-488-5p expression was normal, while P53 KD prevented the effects of the miR-488-5p inhibitor in KYSE-150 cells. Additionally, we found that tumor size was obviously smaller in miR-488-5p overexpression (OE)+ P53 OE mice than miR-488-5p OE mice. Hematoxylin and eosin and immunohistochemistry staining also revealed similar results.

Conclusions: Our results suggest that miR-488-5p promotes ESCC progression by suppressing the P53 pathway. These findings should provide novel ideas for ESCC therapies.

Keywords: KYSE-150; miR-488-5p; esophageal squamous cell carcinoma (ESCC); P53

Submitted Jul 23, 2021. Accepted for publication Sep 17, 2021.

doi: $10.21037 /$ jtd-21-1448

View this article at: https://dx.doi.org/10.21037/jtd-21-1448

(C) Journal of Thoracic Disease. All rights reserved. 


\section{Introduction}

Esophageal carcinoma, which accounts for $1-2 \%$ of all malignant tumors, is one of the most common malignant tumors of the digestive system (1). Among the cases of cancer death worldwide, esophageal carcinoma ranks 6th (2). According to the latest survey data from the International Agency for Research on Cancer, there were 572,000 new esophageal carcinoma cases and 50.8000 deaths in 2018 (3). China has high esophageal carcinoma morbidity and mortality rates (4). Esophageal squamous cell carcinoma (ESCC) and esophageal adenocarcinoma (EAC) are the two main pathological types of esophageal carcinoma (5). There are differences between the two pathological types worldwide (6). In China, esophageal carcinoma mainly originates from the esophageal squamous epithelium, and ESCC is the main histological type, while in Western countries, esophageal carcinoma mainly originates from Barrett's esophagus, and EAC is the main histological type (7). The incidence of esophageal carcinoma may be related to genetic factors, smoking, obesity, and chronic gastro esophageal reflux disease (8).

Micro ribonucleic acids (miRNAs) are small, noncoding RNAs that modulate cell development, invasion, migration, proliferation, and apoptosis. Increasing evidences show that abnormal miRNA expression also has such effects on a variety of tumors, such as lung tumors (9), cervical tumors (10), and gastric tumors (11), and numerous studies have proven that miRNAs influence the tumorigenesis. The deregulation of miRNA expression occurs in tumorigenesis, such as esophageal carcinoma; thus, miRNA expression profiles are important in the early diagnosis of esophageal carcinoma. Liu et al. found that miRNAs can be used as novel potential biomarkers for ESCC diagnosis (12). The role of MiRNAs is similar to that of oncogenes or tumor suppressor genes (13). miR21 has been reported to induce cell growth, migration, and invasion by targeting phosphatase and tensin homolog (PTEN) and programmed cell death 4 (PDCD4) in ESCC $(14,15)$. In ESCC, more miRNAs have been identified that have a tumor suppressor gene function than a oncogene function (16). The overexpression of miR-382 prevents cell migration and invasion through the mammalian target of rapamycin/eukaryotic translation initiation factor $4 \mathrm{E}$ binding protein 1 (mTOR/4E-BP1) signaling pathway in ESCC (16). miR-488-5p, a microRNA, reportedly acts as an inhibitor of androgen receptors in prostate cancer in the earliest research (17). It also has the function of regulating the bone density of women in desperate situations (18). In recent years, it has been discovered that miR-488-5p plays an important role in tumor progression, such as an oncogene in breast cancer (19) and osteosarcoma (20). However, the association of miR-488$5 \mathrm{p}$ with the progression and development of ESCC is still unclear.

Protein 53 (P53) is a tumor suppressor gene containing 11 exons and 10 introns, which can encode proteins containing 393 amino acids (21). Recent studies have shown that P53 is one of the most common genes found in human tumors, and P53 plays an important role in tumor cell apoptosis $(22,23)$. Yao et al. found that the expression of P53 significantly increased in ESCC (24). Additionally, Fagundes et al. found that the expression of P53 was positively correlated with the severity of esophageal carcinoma (25). However, the mechanism by which P53 effects ESCC progression has not yet been fully elucidated.

In this study, we showed that miR-488-5p was upregulated in ESCC, and that miR-488-5p promoted ESCC progression. Further, we found that P53 was a target gene of miR-488-5p in ESCC. We confirmed that miR$488-5$ p promoted ESCC progression by suppressing the P53 pathway. We present the following article in accordance with the ARRIVE reporting checklist (available at https:// dx.doi.org/10.21037/jtd-21-1448).

\section{Methods}

\section{Animal models}

Twenty female Bagg Albino (BALB)/c nu/nu thymusdeficient nude mice weighing $18 \mathrm{~g}$ were obtained from Charles River (Beijing, China). Since gender is not the main factor in the incidence of esophageal cancer (26), we chosed female nude mice. All of the mice were disinfected before being reared. Each cage housed 4 mice in a specificpathogen-free environment. All the mice were kept on a 12-h light-dark cycle. A relative humidity of $50 \% \pm 10 \%$ and a temperature of $22 \pm 2{ }^{\circ} \mathrm{C}$ were maintained. The mice had free access to food (sterilized special mouse pellet feed) and water, and were regularly subjected to ultraviolet irradiation for disinfection. The KYSE- 150 cells $\left[5 \times 10^{5}\right.$ cells in $100 \mu \mathrm{L}$ of phosphate-buffered saline (PBS)] (27) were injected subcutaneously into the back of each 4-week-old mouse. The mice were observed every 2 days for at least 8 weeks, and cervical dislocation was then performed to euthanize the mice. Tumor size was assessed based on volume (length 
$x$ width $\times$ height). The tumor tissue samples obtained from mice were used for the follow-up tests. Experiments were performed under a project license granted by the Institutional Laboratory Animal Care and Use Committee of Hebei Medical University (licenses number: 2018MEC161). All the mice were treated according to national or institutional guidelines for the care and use of animals and the Guide for the Care and Use of Laboratory Animals published by the United States National Institutes of Health (NIH publication, 8th edition, 2011).

\section{Cell culture}

Human normal esophageal epithelial cell line (HEEC) and esophageal cancer cell lines (KYSE-150, TE-10, and TE-15) were purchased from American Type Culture Collection (ATCC, USA). The CRISPR/Cas9 system was used to knockout the TP53 gene in the KYSE-150 cells (i.e., KYSE-150 P53 ${ }^{-/-}$cells). The KYSE-150 P53 ${ }^{-1}$ cells were obtained from Synthgene (Nanjing, China). HEEC, KYSE-150, TE-10, TE-15, and KYSE-150 P53 ${ }^{-/-}$cells were cultured in Roswell Park Memorial Institute (RPMI)-1640 medium supplemented with $10 \%$ fetal bovine serum (FBS) at $37{ }^{\circ} \mathrm{C}$ in an atmosphere containing $5 \%$ carbon dioxide $\left(\mathrm{CO}_{2}\right)$.

\section{Colony formation assay}

The colony formation assay is an effective method for measuring cell viability. The cells in the logarithmic growth phase were inoculated with RPMI-1640 medium at $37{ }^{\circ} \mathrm{C}$ with $5 \% \mathrm{CO}_{2}$. When macroscopic colonies appeared in a culture dish, the culture was terminated. The supernatant was discarded and carefully immersed thrice with phosphate-buffered saline (PBS). $5 \mathrm{~mL}$ of $4 \%$ paraformaldehyde was added to the fixed cells and left for $15 \mathrm{~min}$. The fixative solution was then discarded, an appropriate amount of Giemsa staining solution was added for 10 to $30 \mathrm{~min}$, the staining solution was then slowly washed away with running water, and the plate was left to air dry. The plate was inverted and overlaid using a grid of transparencies. Colonies larger than 10 cells were counted under a microscope (low magnification). Finally, the clone formation rate was calculated.

\section{Cell apoptosis assay}

The cells were collected, resuspended, and centrifuged at $1,000 \mathrm{rpm}$ at room temperature for $5 \mathrm{~min}$. Next, the supernatant was discarded and the sediment was resuspended with $300 \mu \mathrm{L} \times$ binding buffer in each sample, and incubated with $5 \mu \mathrm{L}$ Annexin V-FITC for $10 \mathrm{~min}$, incubated with $5 \mu \mathrm{L}$ Propidium iodide (PI) before detection. Finally, the samples were examined using flow cytometer (Beckman Coulter, CA, USA). The tests were repeated 3 times.

\section{Cell invasion assay}

Transwell chambers with $24-w e l l$ plates were used. The Matrigel was prepared in advance, and $100 \mu \mathrm{L}$ of Matrigel was added to the upper chambers at $37^{\circ} \mathrm{C}$ and left overnight to gel. The Matrigel was reconstructed by adding $200 \mu \mathrm{L}$ of RPMI-1640 medium, and $750 \mu \mathrm{L}$ of culture medium (with serum) to the lower chamber. Next, the cells were plated at $1 \times 10^{5}$ cell $/ \mathrm{mL}$ in the upper chamber of the 24 -well plate chamber, and kept at $37^{\circ} \mathrm{C}$ for $12-16 \mathrm{~h}$. The cells were them treated with $4 \%$ paraformaldehyde for $10 \mathrm{~min}$, and washed thrice with PBS. The cells were treated by GIESMA for $10 \mathrm{~min}$, and then washed 3 times with PBS. Finally, images were captured using a light microscope (Olympus, Tokyo, Japan).

\section{Cell migration assay}

A volume of $600 \mathrm{~mL}$ of RPMI-1640 medium containing $10 \%$ FBS was added to the lower Transwell chamber, and $200 \mathrm{~mL}$ of cell suspension was added to the upper chamber and left for 24 hours. Next, the liquid was removed from the upper and lower chambers, and the cells were then treated with $4 \%$ paraformaldehyde for $30 \mathrm{~min}$, and washed with PBS. Finally, the cells were treated with $600 \mu \mathrm{L}$ of $0.1 \%$ crystal violet for $10 \mathrm{~min}$ and then washed with PBS. The cells were observed under a microscope (Olympus, Tokyo, Japan) and counted.

\section{HE staining}

The tumors were separated into NC, miR-488-5p OE, miR-488-5p KD, and miR-488-5p OE + P53 OE groups. The sliced tumors were dewaxed and hydrated, and the slices were then incubated in hematoxylin solution for 5$20 \mathrm{~min}$ before being washed with water. Next, the slices were differentiated by differentiation fluid for $30 \mathrm{sec}$ and then soaked in water for $15 \mathrm{~min}$. The slices were then incubated in eosin solution for 10-50 sec. Finally, the slices 
were dehydrated, transparentized, sealed, and observed under a microscope (Olympus, Tokyo, Japan).

\section{Immunobistochemistry staining}

The sliced tumors were dewaxed, hydrated, and incubated in $3 \%$ hydrogen peroxide $\left(\mathrm{H}_{2} \mathrm{O}_{2}\right)$ for $10 \mathrm{~min}$ to block endogenous peroxidase activity, and then washed with PBS. The slices were incubated with primary antibody (1:100) overnight at $4{ }^{\circ} \mathrm{C}$, and then incubated with secondary antibodies at room temperature for $1-2 \mathrm{~h}$, washed with $1 \times$ Tris-buffered saline tween (TBST) and incubated in diaminobenzidine chromogen for $5 \mathrm{~min}$. The nucleus was counterstained with 4',6-diamidino-2-phenylindole (DAPI). The slices were observed under a microscope (Olympus, Tokyo, Japan).

\section{Western blot}

Protein from the tumors or cells was isolated using cell lysis buffer (Thermo Fisher, MA, USA). Next, the protein was separated using $10 \%$ sodium dodecyl sulphatepolyacrylamide gel electrophoresis (SDS-PAGE) and transferred to polyvinylidene fluoride or polyvinylidene difluoride (PVDF) membranes. Next, the membranes were incubated with $0.5 \%$ bovine serum albumin for $1 \mathrm{~h}$ at room temperature and then washed in PBS. The membranes were then incubated with primary antibodies $(1: 1,000)$ at $4{ }^{\circ} \mathrm{C}$ overnight. They were then washed and incubated in secondary antibody and kept at room temperature for $1-2 \mathrm{~h}$. Finally, the bands were evaluated using scanning densitometry through enhanced chemiluminescence (ECL; Thermo Fisher, MA, USA). Actin served as the internal control.

\section{qRT-PCR}

RNAs from the cell cultures were isolated using TRIzol reagent (Invitrogen, Shanghai, China) in accordance with the manufacturer's instructions. Reverse transcription of the extracted RNA that was converted into complementary deoxyribonucleic acid (cDNA) was conducted using the HiScript ${ }^{\circledR}$ III 1st Strand cDNA Synthesis Kit (Vazyme, Nanjing, China). MiR-488-5p expression was analyzed on a reverse transcription polymerase chain reaction (RTPCR) detection system (Analytic, Jena, Germany) and quantified using the $\triangle \Delta C T$ method with the following reverse transcription primers: 5'-GTCGTATCCAGTGCA
GGGTCCGAGGTATTCGCACTGGATACGACGACC AA-3'; FP: 5'-GCGATTGAAAGGCTATTTC-3'; and RP: 5'-AGTGCAGGGTCCGAGGTATT-3'.

\section{Luciferase reporter assay}

The putative binding sites of miR-488-5p on the P53 3'-untranslated region (UTR) were predicted by TargetScan7.2. pMIR-P53 3'-UTR-WT, and pMIR-P53 3'-UTR-Mut luciferase reporter plasmids were purchased from Genechem (Shanghai, China). The sequences of TP53 (position 784-804) that bind to miR-488-5p were partly mutated and inserted into the reporter plasmid to identify the binding specificity. The miR-488 mimic and its control were transfected into cells. After $48 \mathrm{~h}$ of transfection, the relative luciferase activities of the cells were measured using the Dual-Glo Luciferase Assay System (Promega, Shanghai, China) in accordance with the manufacturer's introductions. The Renilla signal was used to normalize luciferase activity.

\section{Statistical analysis}

The data are presented as mean \pm standard deviation. The statistical analyses included a 1-way analysis of variance followed by Bonferroni's multiple comparison test (for comparisons of more than two groups) or the Student's $t$-test (for comparisons of two groups) (GraphPad Prism). A P value $<0.05$ was considered statistically significant.

\section{Results}

\section{miR-488-5p promotes the development of ESCC in vitro}

As Figure $1 A$ shows, miR-488-5p expression was significantly more upregulated in KYSE-150 group than the HEEC group. The KYSE-150 cells were transfected with the miR-488-5p inhibitor, and the transfection efficiency was confirmed by the quantitative RT-PCR assay (see Figure 1B). First, the colony formation assays and cell apoptosis detection results indicated that compared to the KYSE-150+NC group, the miR-488-5p inhibitor group showed decreased cell viability and increased cell apoptosis (see Figure 1C,1D). Additionally, the miR-488-5p inhibitor group showed less cell migration than the KYSE-150+NC group (see Figure $1 E$ ). In addition, cell invasion was also inhibited by the miR-488-5p inhibitor (see Figure $1 F$ ). These results indicate that $\mathrm{miR}-488-5 \mathrm{p}$ could promote ESCC progression in KYSE-150 cells. 

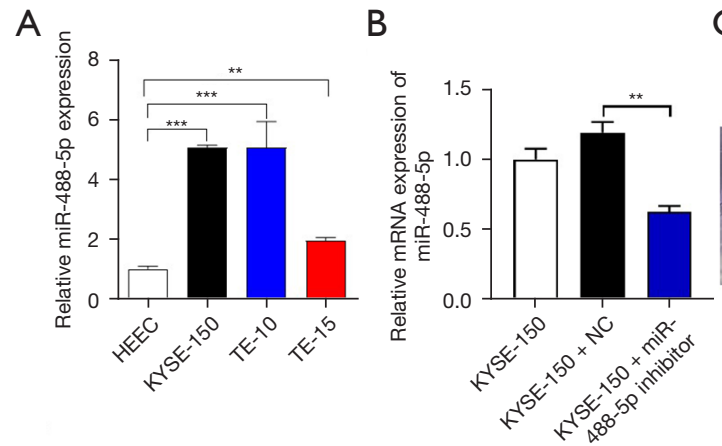

C
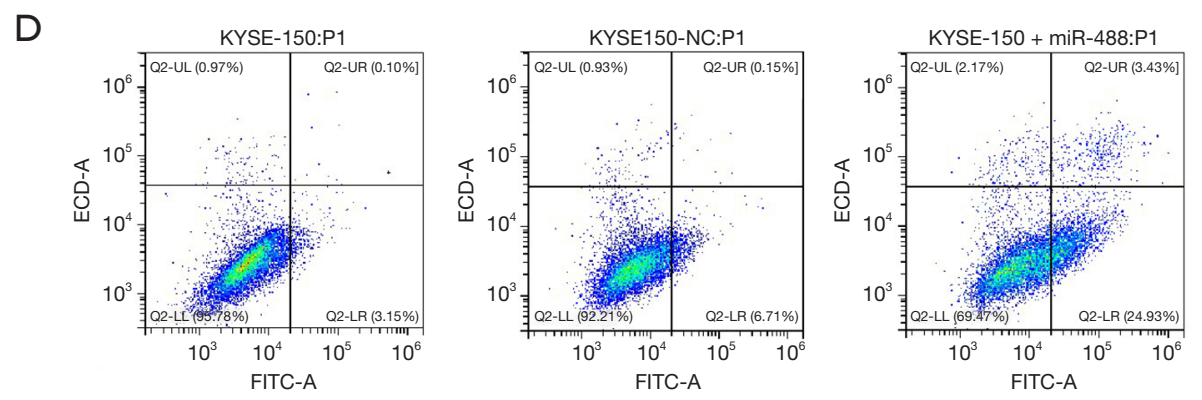

$E$
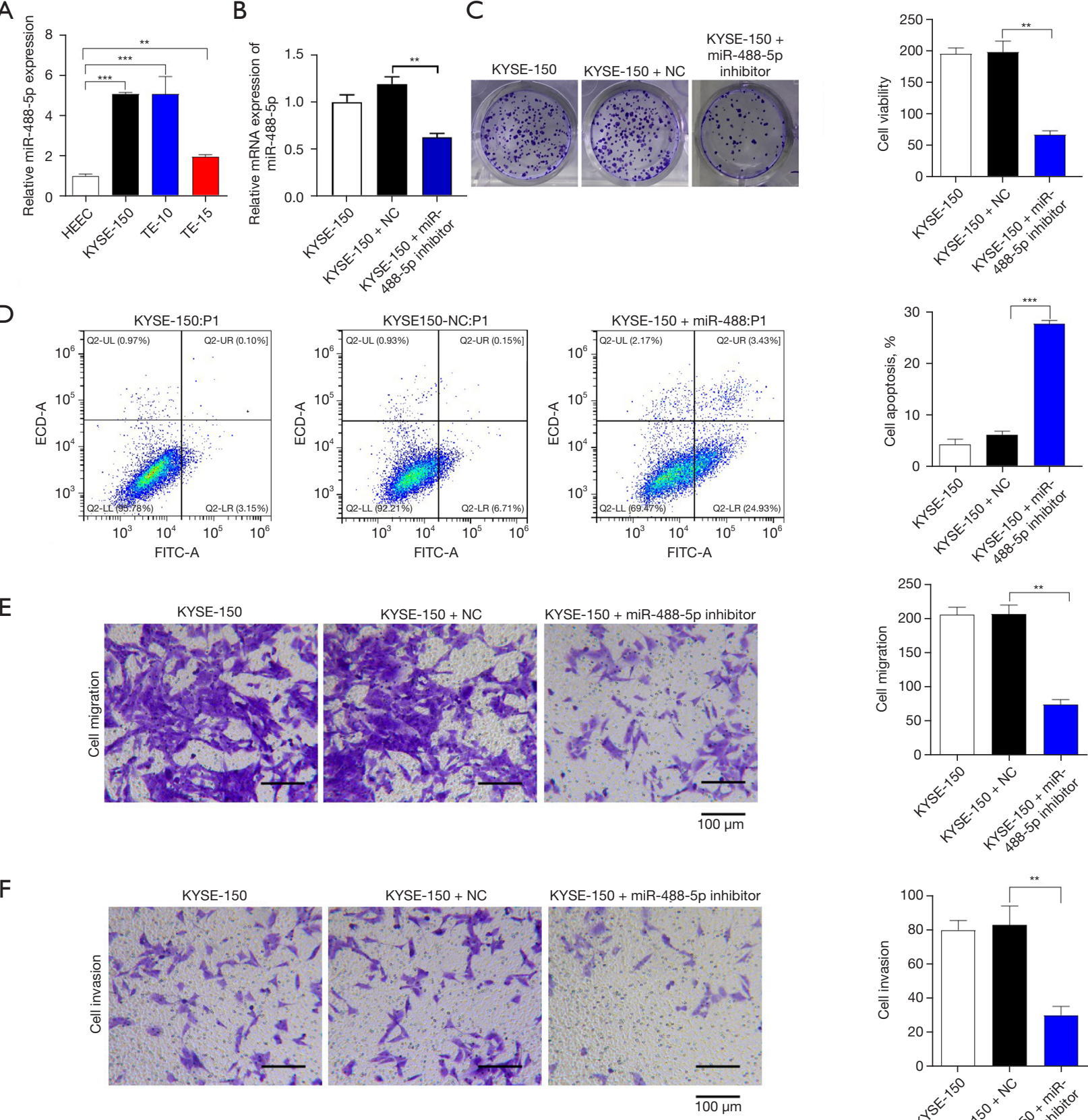

$\mathrm{F}$
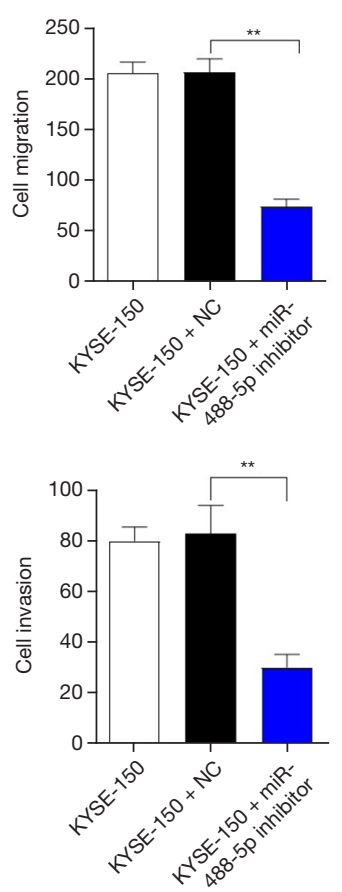

Figure 1 miR-488-5p promotes the development of ESCC in vitro. KYSE-150 cells were treated with the miR-488-5p inhibitor for $48 \mathrm{~h}$. (A) Relative values of miR-488-5p mRNA levels in the HEEC, KYSE-150, TE-10, and TE-15 groups. (B) The transfection efficiency of the miR-488-5p inhibitor was confirmed by a qRT-PCR assay. (C) A colony formation assay showed cell viability. (D) A flow cytometry assay detected cell apoptosis. (E,F) A Transwell assay assessed cell migration and cell invasion. Cells were stained with Giemsa stain and were observed under light microscopy ( $\times 400$ magnification). Values are mean \pm standard error, compared to the KYSE-150 group, ${ }^{* *} \mathrm{P}<0.01$, ${ }^{* * *} \mathrm{P}<0.001, \mathrm{n}=3$ per group. 
A
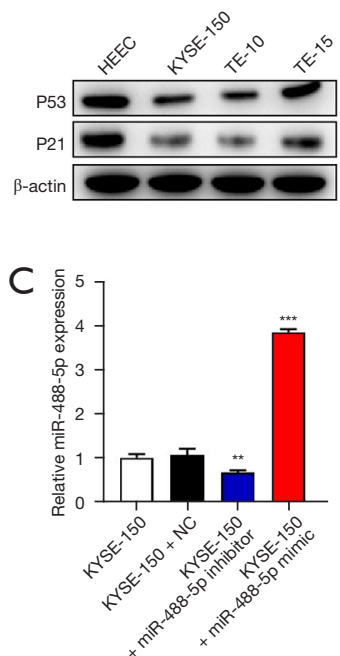

$E$

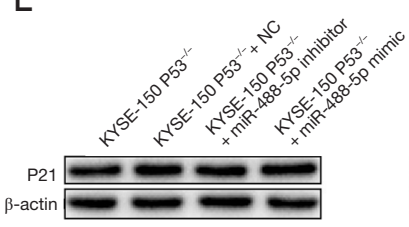

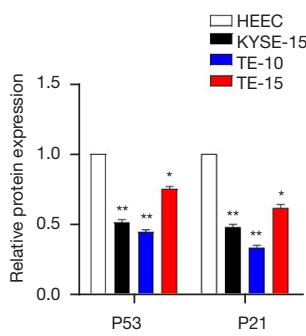

B

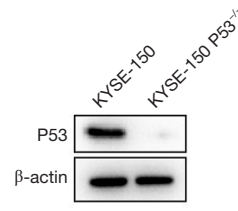

D
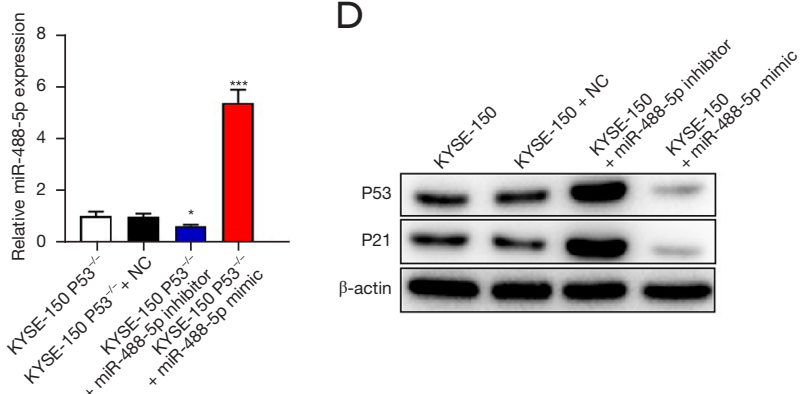

$\mathrm{F}$

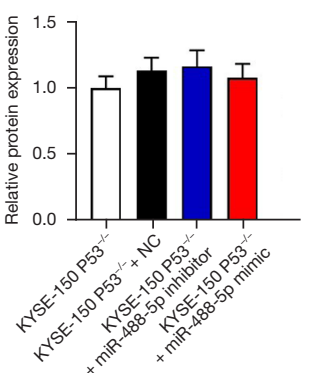

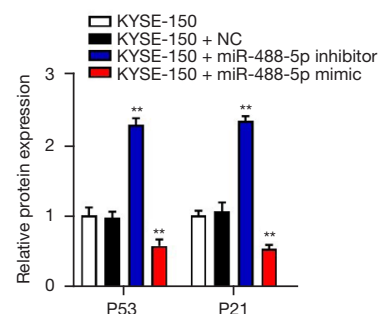

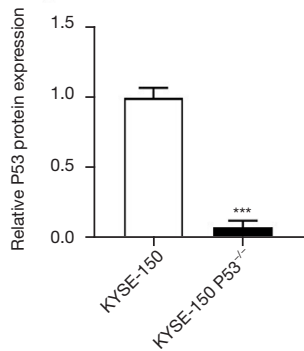

G

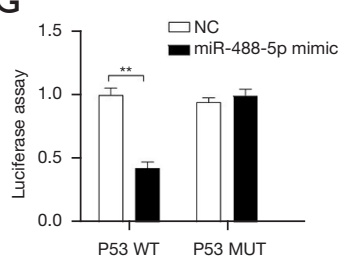

Figure 2 P53 is a target gene of miR-488-5p. KYSE-150 cells were treated with the miR-488-5p inhibitor for $48 \mathrm{~h}$. (A) A Western blot assay detected P53 and P21 protein expression in the HEEC, KYSE-150, TE-10 and TE-15 groups. (B) A Western blot assay detected P53 protein expression in the KYSE-150 P53 $3^{-1-}$ cells. The KYSE-150 cells and KYSE-150 P53 ${ }^{-1-}$ cells were treated with the miR-488-5p inhibitor or the mimic for $48 \mathrm{~h}$. (C) The transfection efficiency of the miR-488-5p inhibitor or the mimic in the KYSE-150 cells or KYSE$150 \mathrm{P}_{53^{-1-}}$ cells was confirmed by a qRT-PCR assay. (D) A Western blot assay detected P53 and P21 protein expression in the KYSE-150 cells. (E) A Western blot assay detected P21 protein expression in the KYSE-150 P53 $3^{-/-}$cells. (F,G) A luciferase reporter assay was performed at $48 \mathrm{~h}$ after transfection KYSE-150 with luciferase reporter plasmid containing the wild type or mutant form of P53 3'-UTR along with the control mimic or the miR-488-5p mimic. Cells were stained with Giemsa stain and were observed under light microscopy $(\times 400$ magnification). Values are mean \pm standard error, compared to the HEEC, KYSE-150, or KYSE-150 P5 $3^{-1-}$ groups. *, $\mathrm{P}<0.05$; **, $\mathrm{P}<0.01$; ***, $\mathrm{P}<0.001 . \mathrm{n}=3$ per group.

\section{$P 53$ is a target gene of $m i R-488-5 p$}

We then explored the relationship between miR-488$5 \mathrm{p}$ and P53 in ESCC. The Western blot showed that the levels of $\mathrm{P} 53$ and $\mathrm{P} 21$ protein were more downregulated in KYSE-150 group than the HEEC group (see Figure 2A). We generated TP53 knockout cells in KYSE-150. The Western blot assay confirmed that the protein expression of P53 was markedly decreased in KYSE-150 P53 $3^{-/}$cells (see Figure 2B). We then treated KYSE-150 cells or KYSE-150
P53 ${ }^{-/-}$cells with the miR-488-5p inhibitor and miR-488-5p mimic for $48 \mathrm{~h}$. The RT-qPCR assay showed that miR-488$5 p$ inhibitor suppressed miR-488-5p expression, while the miR-488-5p mimic significantly upregulated miR-488-5p in the KYSE-150 and KYSE-150 P53 $3^{--}$cells (see Figure 2C). The MiR-488-5p inhibitor obviously increased P53 and P21 expression, while the miR-488-5p mimic decreased P53 and P21 expression (see Figure 2D). However, the miR-488$5 \mathrm{p}$ inhibitor and mimic had no function on P21 expression in KYSE-150 P53 ${ }^{-/}$cells (see Figure $2 E$ ). Next, the online 
TargetScan7.2 database was used to predict the target of miR-488-5p. We found that P53 might be a potential target of miR-488-5p; a promising binding site between miR-488$5 \mathrm{p}$ and P53 is shown in Figure $2 F$. To confirm whether miR488-5p affected P53 expression, the luciferase reporter assay was performed. The luciferase activity was significantly more decreased following co-transfection with pMIR-P53 3'-UTR-WT and miR-488-5p than co-transfection with pMIR-P53 3'-UTR-Mut and miR-488-5p. These results indicate that miR-488-5p can specifically bind to the $3^{\prime}-$ UTR of P53, and negatively regulate the P53 expression (see Figure 2G).

\section{miR-488-5p facilitates ESCC by P53 inbibition in vitro}

To confirm whether miR-488-5p promotes ESCC via the P53 pathway, we treated KYSE-150 cells with miR$488-5 p$ mimic or miR-488-5p inhibitor for 48 h. Colony formation assay demonstrated that compared to the miR488-5p mimic, miR-488-5p inhibitor decreased cell viability in KYSE-150 cells (see Figure 3A). Similarly, apoptosis detection suggested that cell apoptosis was upregulated by miR-488-5p inhibitor compared to miR-488-5p mimic in KYSE-150 cells (see Figure 3B). In addition, we also found that cell migration (see Figure $3 C$ ) and cell invasion (see Figure 3D) were also more inhibited by miR-488-5p inhibitor than the miR-488-5p mimic in KYSE-150 cells. As a comparison, KYSE-150 P $53^{-/-}$cells were also treated with the miR-488-5p mimic or miR-488-5p inhibitor for $48 \mathrm{~h}$. There were no significant differences in cell viability (see Figure 3E), apoptosis (see Figure 3F), cell migration (see Figure 3G), and invasion (see Figure $3 H$ ) between the P53 deletion KYSE-150 cells treated with the miR-488$5 \mathrm{p}$ inhibitor and the miR-488-5p mimic. These results confirmed that P53 is an important target for miR-488-5p in aggravating the development of ESCC.

\section{miR-488-5p promotes ESCC progression in vivo}

In addition, we also established mice model to determine the effects of miR-488-5p on ESCC in vivo. We found that the miR-488-5p OE-induced ESCC tumor size was reduced by $\mathrm{P} 53 \mathrm{OE}$, and ESCC tumor size was more diminished in the miR-488-5p knockdown (KD) group than the NC group (see Figure 4A,4B). Hematoxylin and eosin (HE) staining revealed similar results (see Figure $4 C$ ). The immunohistochemical expressions of P53 and P21 were decreased after being treated with the miR-488-5p mimic and increased after being treated with the miR488-5p inhibitor. The overexpression of P53 reversed the decreased P53 and P2 1 expression trend caused by miR-488-5p overload. The proliferation of tumors also exhibited the same behavior when assessed using Ki67 immunohistochemical detection (see Figure 4D). The above in vivo experiment results indicated that miR-488-5p promoted ESCC progression by suppressing P53 and P21 expression.

\section{Discussion}

In recent years, miRNAs have been the subject of extensive research worldwide $(28,29)$. More and more evidence has shown that miRNAs are related to a number of types of tumor progression (30). The discovery of miRNA has generated novel ideas for therapeutic targets of ESCC. In the present study, we found that miR-488-5p expression was significantly upregulated in ESCC cells, indicating the potential role of miR-488-5p in ESCC. Thus, we aimed to explore the role and molecular mechanism of miR-488-5p in ESCC.

Previous studies have suggested that the abnormal expression of miR-488-5p is involved in the development of various diseases. For example, miR-488-5p is considered a novel prognostic biomarker for breast cancer (19). Zhou et al. found that transfection of a microRNA-488$5 \mathrm{p}$ inhibitor resulted in an increase in apoptosis and a decrease in proliferation in osteosarcoma (20). Arnold et al. revealed that miR-488-5p suppressed melanoma development (31). It can be seen that miR-488-5p is expressed both as an oncogene and also as a tumor suppressor gene. It is not uncommon for the same miRNA molecule to exhibit different functions under different circumstances. For example, different studies believe that miR-23b-3p has diametrically opposite effects on breast cancer. Pellegrino et al. (32) found that miR-23b-3p regulates focal adhesion, cell spreading, cell-cell junctions and the formation of lamellipodia in breast cancer, and exerts a tumor suppressor effect. Jin et al. (33) believed that miR-23b (by comparing the nucleic acid sequence with the database here is indeed miR-23b-3p) and miR$27 \mathrm{~b}$ gene knockdown can significantly inhibit the growth of breast cancer, by knocking down miR-23b and miR$27 \mathrm{~b}$ can enhance the expression of Nischarin, so miR-23b$3 p$ is considered to be an oncogene. It can be seen that the same miRNA molecule may have different functions in different situations, and even in the same disease, there 

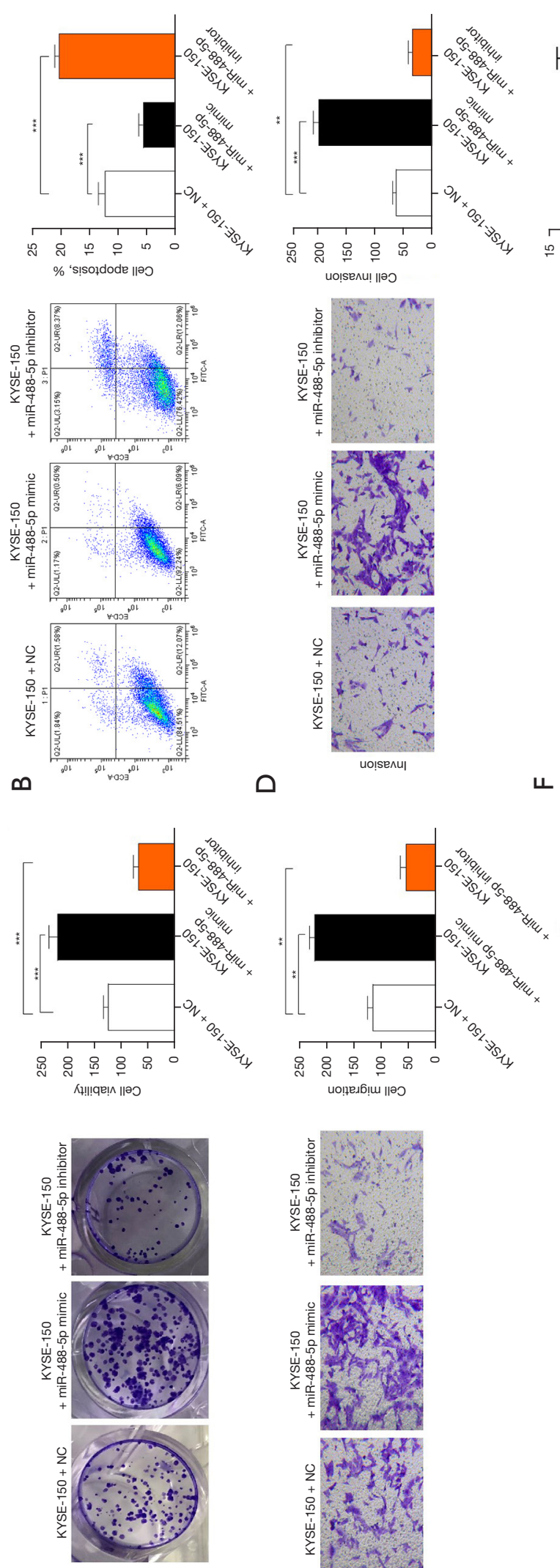

$\varangle$
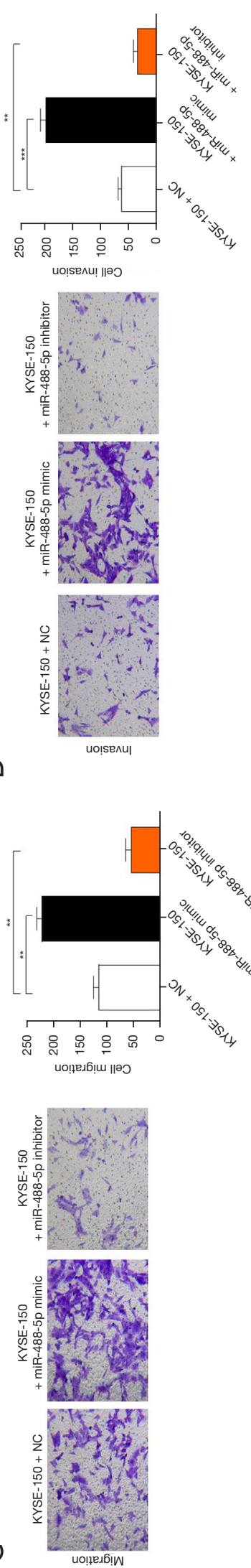

D

丩
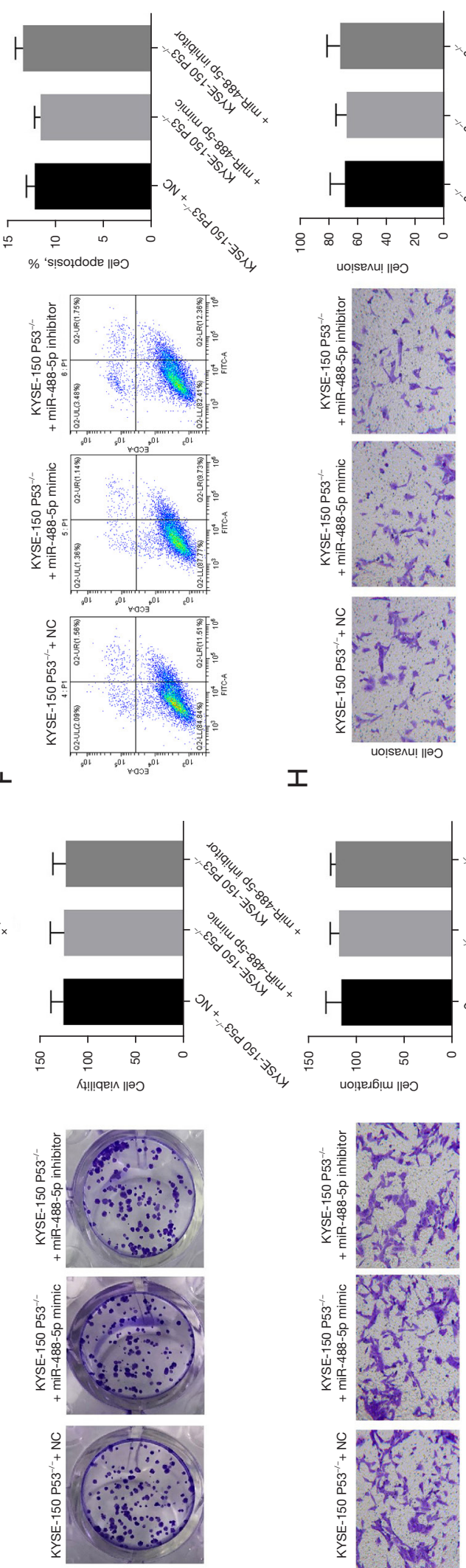

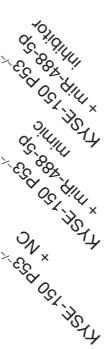

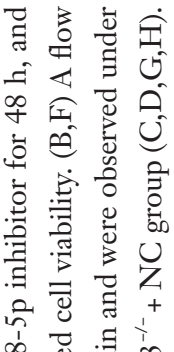

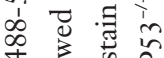

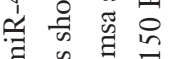

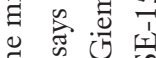

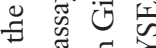

○

.

ह. 유

की

$\infty$ 잉

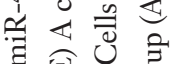

¿

号这

엉

Ð

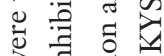

$3 . \Xi \cdot D$

卷 㐘

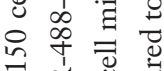

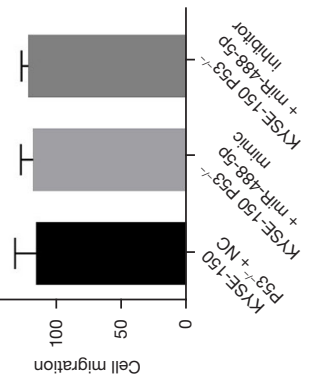

兵角总

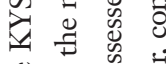

E

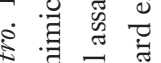

施

₹ $\infty$ त्ञा

: 号

寻角灭

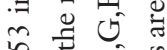

ลิ 1 \&

을

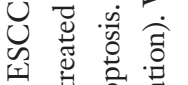

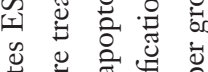

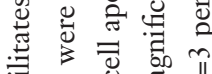

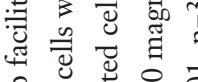

in 1 d

定

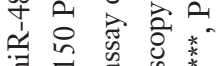

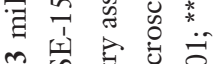

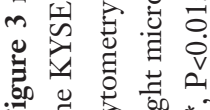


A

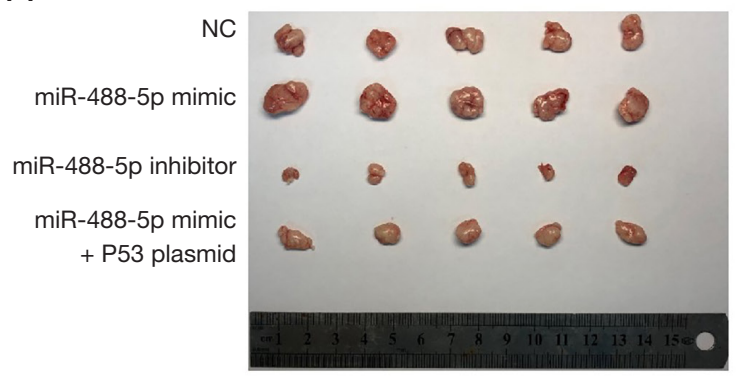

C
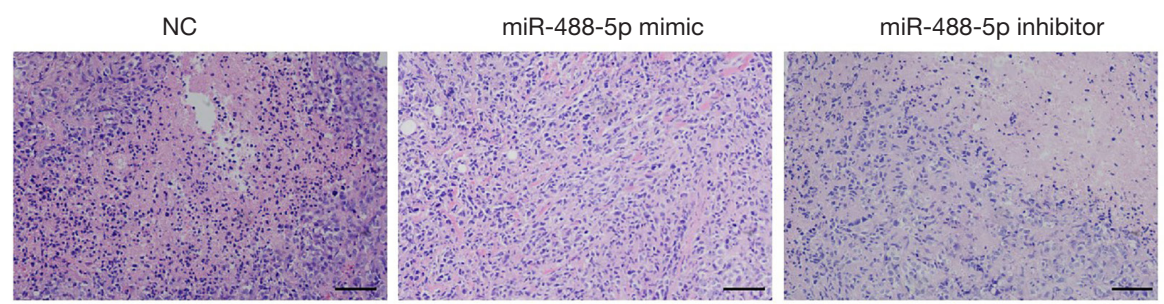

$\mathrm{D}$
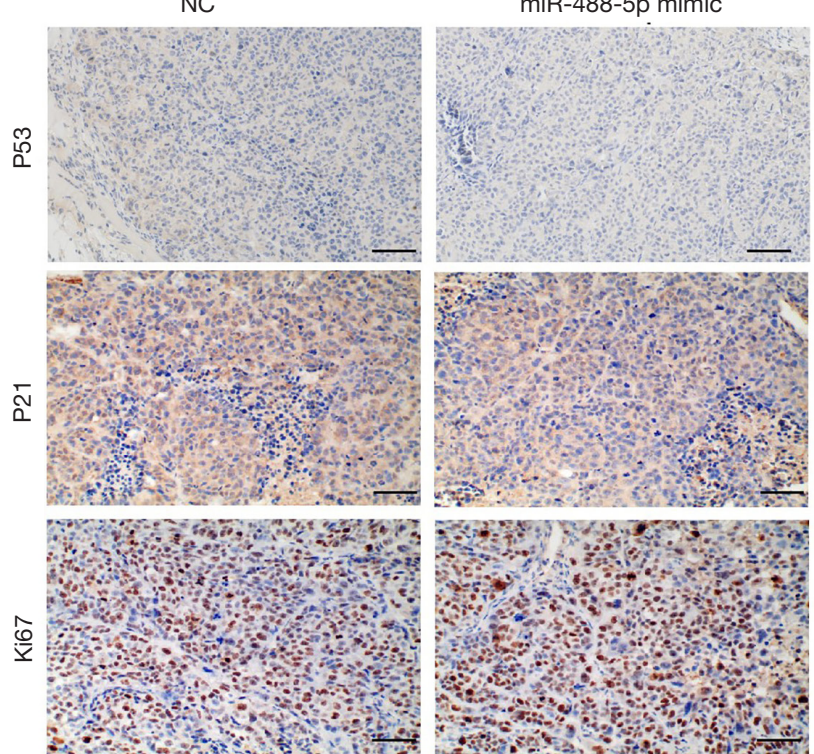

miR-488-5p mimic + P53 plasmid

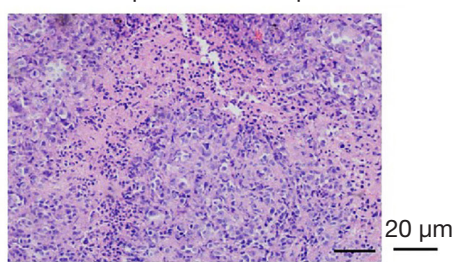

B

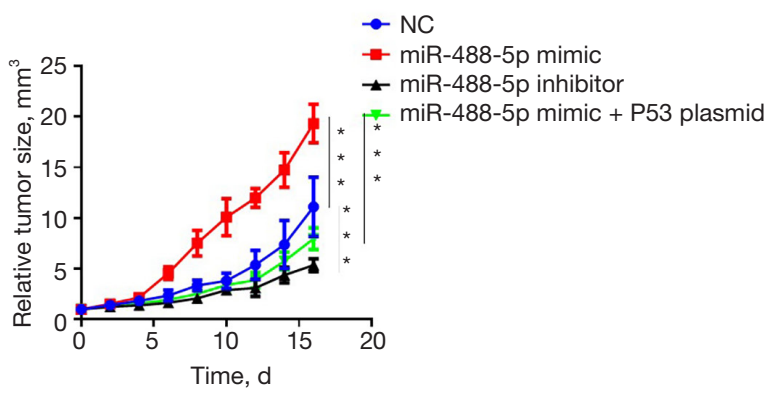

$\underline{20 \mu \mathrm{m}}$

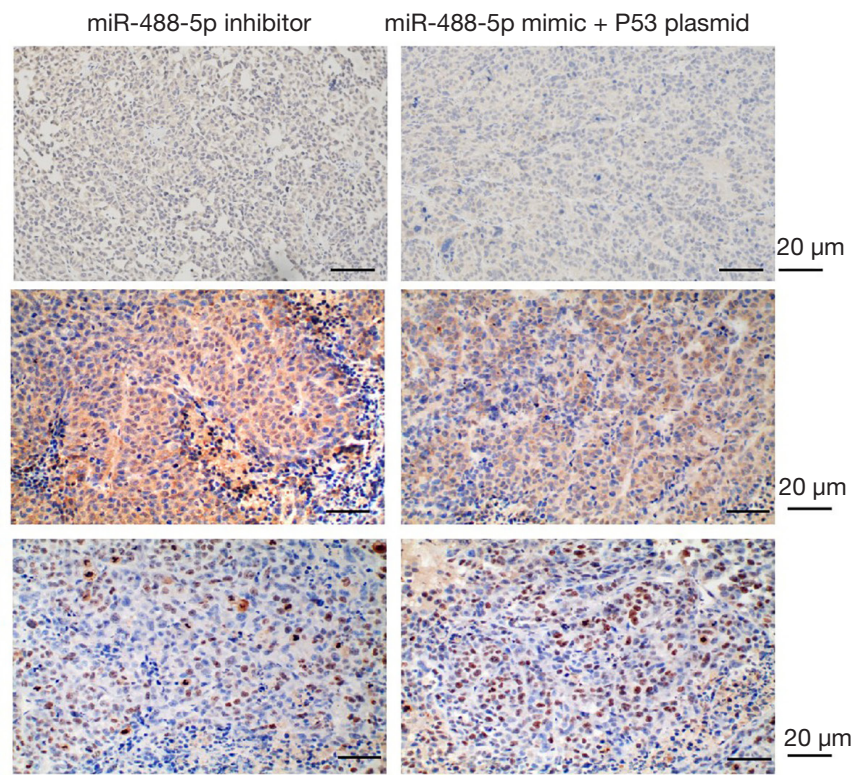

Figure 4 miR-488-5p promotes ESCC in vivo. Tumors were separated in the NC, miR-488-5p OE, miR-488-5p KD, and miR-488-5p OE + P53 OE mice models. (A) Representative images of tumors from the NC, miR-488-5p OE, miR-488-5p KD and miR-488-5p OE + P53 OE groups. (B) Relative tumor size of tumor. (C) Representative images of HE staining of tumors. Bar $=20 \mu \mathrm{m}$. (D) Representative images of immunohistochemical staining for P53, P21 and Ki67 (brown color) in tumors. Bar $=20 \mu \mathrm{m}$. Values are mean \pm standard error, compared to the $\mathrm{NC}$ group. ${ }^{* * *}, \mathrm{P}<0.001 . \mathrm{n}=5$ per group.

may be diametrically opposite manifestations. The specific reasons are still unclear. Additionally, Huang et al. found that miR-488-5p inhibited mycobacterium tuberculosis associated macrophage polarization (34). However, no research had previously been conducted on whether miR$488-5$ p regulated ESCC progression. Our research studied the pathological function of ESCC using KYSE-150 cells. We found that cell viability, invasion, and migration 
were significantly decreased by the miR-488-5p inhibitor compared to NC in KYSE-150 cells, while cell apoptosis was upregulated. These results indicate that miR-488-5p appears to be an oncogene in ESCC.

MiRNA is a key regulator of transcriptional and posttranscriptional gene silencing in tumor development $(28,35)$. Evidence has shown that miRNAs play biological roles by directly binding to the 3'-UTR of mRNAs and thereby suppressing mRNA level. For example, Wang et al. showed that miR-488-3p promoted osteoblast function by targeting the Dickkopf WNT signaling pathway inhibitor 1 (Dkk1) (36). MiR-488-3p was shown to regulate the production of proinflammatory cytokines in acute gouty arthritis by targeting the 3 'UTR of interleukin-1 $\beta$ (IL-1 $\beta$ ) (37). In the present study, we performed a bioinformatics analysis to clarify the mechanism underlying miR-488-5p, and predicted that P53 was a possible target of miR-488-5p. P53 has been reported to inhibit DNA damage and chromosomal aberrations, and thus prevent the transmission of these aberrations to daughter cells (38). P53 is one of the most important tumor suppressor genes and is frequently mutated in human cancers (39). For example, $\mathrm{Xu}$ et al. suggested that the P53 signaling pathway suppressed ESCC cell growth and promoted apoptosis (40). P21 has been shown to be a downstream transactivation target of P53 (41). Further, the level of P21 has been shown to be positively correlated with P53 in ESCC (42). Tang et al. clarified that the upregulation of P53 and P21 increased the survival of patients with ESCC (43). Our research indicated that P53 and P21 levels were decreased in ESCC cell lines.

In addition, miR-488-5p negatively regulated P53 and P21 expression in KYSE-150 cells. Interestingly, miR-488$5 \mathrm{p}$ had no effect on P21 expression in KYSE-150 P53 $3^{-/-}$ cells. We performed a luciferase assay and confirmed that miR-488-5p negatively regulated P53 expression directly by binding to the 3 '-UTR of P53 mRNA. In addition, the promotion effects of the miR-488-5p mimic on cell viability, cell invasion, and cell migration were markedly more inhibited in KYSE- 150 P $53^{-/-}$cells than KYSE-150 cells, and the miR-488-5p inhibitor also produced similar results. We also explored the mechanism of miR-488-5p in vivo, and found miR-488-5p OE-induced ESCC tumor size was reduced by P53 OE, and ESCC tumor size was also significantly diminished in the miR-488-5p knockdown (KD) group than the NC group. HE staining showed similar results. The immunohistochemical expression of
P53 and P21 decreased with miR-488-5p OE treatment, which were prevented by $\mathrm{P} 53 \mathrm{OE}$.

It should be noted that this study had some limitations. For example, drug resistance is the most common challenge that arises in ESCC treatment (44); however, the question of whether miR-488-5p plays a role in promoting drug sensitivity has not yet been investigated. We will explore the function of miR-488-5p in relation to the overexpression and inhibition of miR-488-5p in drug-sensitive ESCC cells in the future.

In conclusion, miR-488-5p promoted ESCC progression by mediating P53 expression. Further analyses of the mechanism of miR-488-5p in ESCC could lead to novel ideas being generated for ESCC treatments.

\section{Acknowledgments}

We would like to thank all the researchers for their contributions.

Funding: This work was supported by the 2020 Hebei Provincial Government-Funded Medical Scientific Research Projects Plan from China (20200229).

\section{Footnote}

Reporting Checklist: The authors have completed the ARRIVE reporting checklist. Available at https://dx.doi. org/10.21037/jtd-21-1448

Data Sharing Statement: Available at https://dx.doi. org/10.21037/jtd-21-1448

Conflicts of Interest: All authors have completed the ICMJE uniform disclosure form (available at https://dx.doi. org/10.21037/jtd-21-1448). The authors have no conflicts of interest to declare.

Ethical Statement: The authors are accountable for all aspects of the work, including ensuring that any questions related to the accuracy or integrity of any part of the work have been appropriately investigated and resolved. Experiments were performed under a project license granted by the Institutional Laboratory Animal Care and Use Committee of Hebei Medical University (licenses number: 2018MEC161). All mice were treated according to national or institutional guidelines for the care and use of animals and the Guide for the Care and Use of Laboratory Animals published by the United States 
National Institutes of Health (NIH publication, 8th edition, 2011).

Open Access Statement: This is an Open Access article distributed in accordance with the Creative Commons Attribution-NonCommercial-NoDerivs 4.0 International License (CC BY-NC-ND 4.0), which permits the noncommercial replication and distribution of the article with the strict proviso that no changes or edits are made and the original work is properly cited (including links to both the formal publication through the relevant DOI and the license). See: https://creativecommons.org/licenses/by-nc-nd/4.0/.

\section{References}

1. Krill T, Baliss M, Roark R, et al. Accuracy of endoscopic ultrasound in esophageal cancer staging. J Thorac Dis 2019;11:S1602-9.

2. Uhlenhopp DJ, Then EO, Sunkara T, et al. Epidemiology of esophageal cancer: update in global trends, etiology and risk factors. Clin J Gastroenterol 2020;13:1010-21.

3. Bray F, Ferlay J, Soerjomataram I, et al. Global cancer statistics 2018: GLOBOCAN estimates of incidence and mortality worldwide for 36 cancers in 185 countries. CA Cancer J Clin, 2018, 68:394-424.

4. Yang X, Zhang T, Yin X, et al. Adult height, body mass index change, and body shape change in relation to esophageal squamous cell carcinoma risk: A populationbased case-control study in China. Cancer Med 2019;8:5769-78.

5. Fatehi Hassanabad A, Chehade R, Breadner D, et al. Esophageal carcinoma: Towards targeted therapies. Cell Oncol (Dordr) 2020;43:195-209.

6. Lordick F, Mariette C, Haustermans K, et al. Oesophageal cancer: ESMO Clinical Practice Guidelines for diagnosis, treatment and follow-up. Ann Oncol 2016;27:v50-7.

7. Yan T, Cui H, Zhou Y, et al. Multi-region sequencing unveils novel actionable targets and spatial heterogeneity in esophageal squamous cell carcinoma. Nat Commun 2019;10:1670.

8. Dhakras P, Uboha N, Horner V, et al. Gastrointestinal cancers: current biomarkers in esophageal and gastric adenocarcinoma. Transl Gastroenterol Hepatol 2020;5:55.

9. Zhang J, Shao W, Zhang Z, Liu D. The rate of miR-146a rs2910164 mutations in patients with lung cancer: a metaanalytic review. Transl Cancer Res 2020;9:6792-800.

10. Wu WK, Lee CW, Cho CH, et al. MicroRNA dysregulation in gastric cancer: a new player enters the game. Oncogene 2010;29:5761-71.

11. Hu X, Schwarz JK, Lewis JS Jr, et al. A microRNA expression signature for cervical cancer prognosis. Cancer Res 2010;70:1441-8.

12. Liu F, Tian T, Xia LL, et al. Circulating miRNAs as novel potential biomarkers for esophageal squamous cell carcinoma diagnosis: a meta-analysis update. Dis Esophagus 2017;30:1-9.

13. Yang $\mathrm{H}, \mathrm{Su} \mathrm{H}, \mathrm{Hu} \mathrm{N}$, et al. Integrated analysis of genomewide miRNAs and targeted gene expression in esophageal squamous cell carcinoma (ESCC) and relation to prognosis. BMC Cancer 2020;20:388.

14. Wang B, Hua P, Zhang L, et al. LncRNA-IUR upregulates PTEN by sponging miR-21 to regulate cancer cell proliferation and apoptosis in esophageal squamous cell carcinoma. Esophagus 2020;17:298-304.

15. Liu T, Liu Q, Zheng S, et al. MicroRNA-21 promotes cell growth and migration by targeting programmed cell death 4 gene in Kazakh's esophageal squamous cell carcinoma. Dis Markers 2014;2014:232837.

16. Feng J, Qi B, Guo L, et al. miR-382 functions as a tumor suppressor against esophageal squamous cell carcinoma. World J Gastroenterol 2017;23:4243-51.

17. Sikand K, Slaibi JE, Singh R, et al. miR 488* inhibits androgen receptor expression in prostate carcinoma cells. Int J Cancer 2011;129:810-9.

18. Coronnello C, Hartmaier R, Arora A, et al. Novel modeling of combinatorial miRNA targeting identifies SNP with potential role in bone density. PLoS Comput Biol 2012;8:e1002830.

19. Masuda T, Shinden Y, Noda M, et al. Circulating PremicroRNA-488 in Peripheral Blood Is a Potential Biomarker for Predicting Recurrence in Breast Cancer. Anticancer Res 2018;38:4515-23.

20. Zhou C, Tan W, Lv H, et al. Hypoxia-inducible microRNA-488 regulates apoptosis by targeting Bim in osteosarcoma. Cell Oncol (Dordr) 2016;39:463-71.

21. Singh M, Aggarwal S, Mohanty AK, et al. Isolation, characterization and functional analysis of full length $\mathrm{p} 53$ cDNA from Bubalus bubalis. Gene 2015;568:146-54.

22. Duffy MJ, Synnott NC, Crown J. Mutant p53 as a target for cancer treatment. Eur J Cancer 2017;83:258-65.

23. Lacroix M, Riscal R, Arena G, et al. Metabolic functions of the tumor suppressor p53: Implications in normal physiology, metabolic disorders, and cancer. Mol Metab 2020;33:2-22.

24. Yao J, Zhang H, Li H, et al. P53-regulated lncRNA uc061hsf. 1 inhibits cell proliferation and metastasis in 
human esophageal squamous cell cancer. IUBMB Life 2020;72:401-12.

25. Fagundes RB, Mello CR, Tollens P, et al. p53 protein in esophageal mucosa of individuals at high risk of squamous cell carcinoma of the esophagus. Dis Esophagus 2001;14:185-90.

26. Sharkey FE, Fogh J. Metastasis of human tumors in athymic nude mice. Int J Cancer 1979;24:733-8.

27. Ip JC, Ko JM, Yu VZ, et al. A versatile orthotopic nude mouse model for study of esophageal squamous cell carcinoma. Biomed Res Int. 2015;2015:910715.

28. Correia de Sousa M, Gjorgjieva M, Dolicka D, et al. Deciphering miRNAs' Action through miRNA Editing. Int J Mol Sci 2019;20:6249.

29. Liu B, Li J, Cairns MJ. Identifying miRNAs, targets and functions. Brief Bioinform 2014;15:1-19.

30. Orso F, Quirico L, Dettori D, et al. Role of miRNAs in tumor and endothelial cell interactions during tumor progression. Semin Cancer Biol 2020;60:214-24.

31. Arnold J, Engelmann JC, Schneider N, et al. miR488-5p and its role in melanoma. Exp Mol Pathol 2020;112:104348.

32. Pellegrino L, Stebbing J, Braga VM, et al. miR-23b regulates cytoskeletal remodeling, motility and metastasis by directly targeting multiple transcripts. Nucleic Acids Res 2013;41:5400-12.

33. Jin L, Wessely O, Marcusson EG, et al. Prooncogenic factors miR-23b and miR-27b are regulated by Her2/ Neu, EGF, and TNF- $\alpha$ in breast cancer. Cancer Res 2013;73:2884-96.

34. Huang Z, Yao F, Liu J, et al. Up-regulation of circRNA-0003528 promotes mycobacterium tuberculosis associated macrophage polarization via down-regulating miR-224-5p, miR-324-5p and miR-488-5p and up- regulating CTLA4. Aging (Albany NY) 2020;12:25658-72.

35. Ali Syeda Z, Langden SSS, Munkhzul C, et al. Regulatory Mechanism of MicroRNA Expression in Cancer. Int J Mol Sci 2020;21:1723.

36. Wang F, Hu XY, Cao C, et al. MiR-488 promotes fracture healing by targeting DKK1. Eur Rev Med Pharmacol Sci 2018;22:8965-72.

37. Zhou W, Wang Y, Wu R, et al. MicroRNA-488 and -920 regulate the production of proinflammatory cytokines in acute gouty arthritis. Arthritis Res Ther 2017;19:203.

38. Williams AB, Schumacher B. p53 in the DNA-DamageRepair Process. Cold Spring Harb Perspect Med 2016;6:a026070.

39. Kadosh E, Snir-Alkalay I, Venkatachalam A, et al. The gut microbiome switches mutant p53 from tumour-suppressive to oncogenic. Nature 2020;586:133-8.

40. Xu Y, Zhu K, Chen J, et al. SASS6 promotes proliferation of esophageal squamous carcinoma cells by inhibiting the p53 signaling pathway. Carcinogenesis 2021;42:254-62.

41. Kim EM, Jung CH, Kim J, et al. The p53/p21 Complex Regulates Cancer Cell Invasion and Apoptosis by Targeting Bcl-2 Family Proteins. Cancer Res 2017;77:3092-100.

42. Taghavi N, Biramijamal F, Sotoudeh M, et al. Association of p53/p21 expression with cigarette smoking and prognosis in esophageal squamous cell carcinoma patients. World J Gastroenterol 2010;16:4958-67.

43. Tang H, Wu Y, Liu M, et al. SEMA3B improves the survival of patients with esophageal squamous cell carcinoma by upregulating p53 and p21. Oncol Rep 2016;36:900-8.

44. Su D, Zhang D, Jin J, et al. Identification of predictors of drug sensitivity using patient-derived models of esophageal squamous cell carcinoma. Nat Commun 2019;10:5076.

(English Language Editor: L. Huleatt)
Cite this article as: Su C, Liu W, Jiang T, Liu J. miR-488-5p promotes esophageal squamous cell carcinoma progression by suppressing the P53 pathway. J Thorac Dis 2021;13(9):55345545. doi: $10.21037 /$ jtd-21-1448 